\title{
Manifestação Otológica Localizada em Paciente com Granulomatose de Wegener
}

\section{Localized Otologic Manifestation in a Patient with Wegener's Granulomatosis}

\author{
Márcia Regina Rosa Scalcon ${ }^{(1)}$, Ivânio Alves Pereira ${ }^{(2)}$, Acir Rachid Filho ${ }^{(3)}$, Eduardo dos Santos Paiva ${ }^{(3)}$
}

\section{RESUMO}

A granulomatose de Wegener (GW) é uma vasculite granulomatosa multissistêmica rara, idiopática, que acomete vasos de pequeno e médio calibres, e que classicamente envolvem os tratos respiratórios superior, inferior e rins. Na forma limitada da doença, diversos sítios anatômicos podem ser afetados isoladamente. Foi descrito o caso de uma paciente jovem, que iniciou o acometimento da GW por dispnéia, estridor laríngeo e hipacusia. A investigação evidenciou o acometimento localizado na glote, e envolvimento da membrana timpânica comprovado por biópsia. A paciente obteve resposta com o tratamento instituído, mas permaneceu com seqüelas otológicas. Revisa-se neste artigo algumas das manifestações focais da GW, salientando especialmente as manifestações otológicas.

Palavras-chave: granulomatose de Wegener, surdez, membrana timpânica.

\section{INTRODUÇÃO}

A granulomatose de Wegener (GW) é uma doença multissistêmica rara, de etiologia desconhecida, descrita inicialmente por Klinger, em 1931, e detalhada por Wegener, em 1936 e $1939^{(1,2)}$.

Histologicamente, a doença é caracterizada pelo processo granulomatoso, necrotizante e ulcerado do tratos respiratórios superior e inferior e de outros órgãos internos; além de vasculite necrotizante e glomerulonefrite focal. Essas três alterações patológicas são determinadas como tríade clássica ${ }^{(3)}$. Manifestações diversas da tríade clássica podem ocorrer, dificultando o diagnóstico. Os exames complementares, como VHS, c-ANCA e histologia, além de alto índice de suspeição, são imprescindíveis no diagnóstico dessa entidade clínica.

\begin{abstract}
Wegener's granulomatosis (WG) is a rare, idiopathic granulomatous vasculitis, affecting small and medium-sized vessels, classically involving upper and lower respiratory tracts and kidneys. In the limited form of the disease, several other sites can be affected. We describe the case of a young woman who presented her disease with dyspnea, laryngeal stridor and hearing loss. Investigation revealed localized glottis involvement and a tympanum biopsy confirmed granulomatous vasculitis. The patient had a partial response with the treatment, with residual deafness. We performed a review concerning the focal manifestations of $W G$, focusing in the ear involvement.
\end{abstract}

Keywords: Wegener's granulomatosis, deafness, tympanum.

A doença pode ser classificada na sua apresentação inicial como forma limitada ou generalizada, sendo que aquilo que define a doença limitada é a ausência do comprometimento renal $^{(4)}$. As manifestações otológicas, como sintomas iniciais da GW, ocorrem em $20 \%$ a $25 \%$ dos pacientes e o diagnóstico pode ser difícil diante desse acometimento locorregional ${ }^{(5)}$.

\section{RELATO DE CASO}

Paciente do sexo feminino, branca, 21 anos, previamente hígida, iniciou com dispnéia aos médios esforços, estridor laríngeo e hipoacusia à direita seis anos antes da avaliação atual, sendo tratada com antibióticos sem sucesso. $\mathrm{Na}$ avaliação laboratorial apresentava hemograma, parcial de urina, função renal e hepática normais. A velocidade de hemossedimentação (VHS) era de $6 \mathrm{~mm}$; proteína C reativa

Recebido em 10/4/2008. Aprovado, após revisão, em 17/7/2008. Declaramos a inexistência de conflitos de interesse

1. Especializanda em Reumatologia do Hospital de Clínicas da Universidade Federal do Paraná (UFPR).

2. Professor-assistente da disciplina de Reumatologia da Universidade Federal de Santa Catarina (UFSC).

3. Professor-assistente da disciplina de Reumatologia da UFPR.

Endereço para correspondência: Márcia Regina Rosa Scalcon, Rua Presidente Carlos Cavalcanti, 649/51, 80510-040, Curitiba, PR, e-mail: marciascalcon@terra.com.br 
inferior a 6,0 (referência normal $<6,0$ ); sorologias virais, fator reumatóide, fator antinuclear e anticorpos anticitoplasma de neutrófilos (ANCA) negativos. Foi submetida à ressonância nuclear magnética (RNM) de crânio e região cervical, que demonstrou otite crônica à direita e estenose subglótica, respectivamente. A avaliação otorrinolaringológica com obtenção de material de biópsia de membrana timpânica evidenciou inflamação granulomatosa com vasculite de pequenos vasos, sugerindo GW.

$\mathrm{Na}$ época foi optado pelo tratamento com prednisona oral $1 \mathrm{mg} / \mathrm{kg} /$ dia com redução gradual associada à ciclofosfamida oral durante dois anos. A paciente apresentou melhora sintomática, porém com seqüela auditiva leve à direita. Passados três anos sem acompanhamento médico, mantendo-se assintomática, a paciente apresentou sintomas constitucionais, seguidos novamente de dispnéia aos médios esforços, estridor, rouquidão e piora da acuidade auditiva à direita, sendo então encaminhada para o Serviço de Reumatologia do Hospital de Clínicas da Universidade Federal do Paraná para reavaliação do quadro.

Durante a internação foi submetida à nova RNM de crânio que evidenciou velamento da mastóide direita por processo inflamatório e/ou infeccioso, a tomografia de tórax foi normal, o sedimento urinário inocente e a endoscopia peroral com espessamento subglótico significativo. Novamente funções renal e hepática, sorologias e c-ANCA foram negativos. As provas de atividade inflamatória foram, respectivamente, VHS $16 \mathrm{~mm}$ e proteína C reativa de 0,65.

Com base no diagnóstico prévio de GW, mesmo com VHS normal e c-ANCA negativo, foi considerada a probabilidade de recidiva da doença e tratada, como no quadro inicial, com prednisona e ciclofosfamida oral, obtendo-se, novamente, boa resposta terapêutica, porém mantendo hipoacusia residual.

\section{DISCUSSÃO}

Os distúrbios auditivos podem ocorrer em qualquer fase da evolução da GW, podendo acometer a orelha externa, média ou interna ${ }^{(6)}$. A orelha média é o local mais freqüentemente acometido ${ }^{(6)} \mathrm{e}$ o déficit otológico dominante nesses pacientes é a perda auditiva condutiva, decorrente do envolvimento granulomatoso nasofaríngeo, disfunção secundária da tuba auditiva e otite média serosa ${ }^{(7,8)}$. Os sinais clínicos incluem a presença de tímpano côncavo brilhante, com ou sem vasos superficiais, e aparência amarelada ${ }^{(7)}$. Em casos raros, a lesão granulomatosa pode ser vista por meio da membrana timpânica ${ }^{(8)}$. A paralisia facial decorrente do envolvimento do sétimo par craniano ocorre em uma pequena porcentagem de pacientes ${ }^{(8)}$. Alguns podem também sofrer de otite média purulenta aguda ou crônica, visto que esta, muitas vezes, pode levar à suspeita de GW por causa do seu caráter refratário ou recorrente ${ }^{(6)}$.

A perda auditiva neurossensorial ocorre em virtude do envolvimento da orelha interna, porém é menos comum. A sintomatologia inclui hipoacusia uni ou bilateral, geralmente assimétrica, acompanhada ou não de outros sintomas, como vertigem, tinnitus e plenitude auricular ${ }^{(6)}$. Padrões mistos de perda auditiva condutiva e neurossensorial são vistos freqüentemente ${ }^{(7)}$. A associação entre hipoacusia $e$ níveis séricos de ANCA não foi comprovada.

Revisões otorrinolaringológicas citam o uso do c-ANCA para o diagnóstico precoce na forma limitada da $\mathrm{GW}^{(2,9)}$, porém mais de $40 \%$ dos pacientes com as formas limitadas da doença podem ser ANCA negativos ${ }^{(10)}$, enfatizando que a pedra angular do diagnóstico permanece sendo a combinação das características clínicas e os achados histológicos no órgão envolvido ${ }^{(10)}$.

O prognóstico da audição pode ser reservado, quando o tratamento apropriado não é instituído em estágios precoces da doença ${ }^{(11)}$. Portanto, a GW deve ser incluída no diagnóstico diferencial dos casos atípicos de estados inflamatórios do ouvido(11).

Um regime de três meses de prednisona $(1 \mathrm{mg} / \mathrm{kg} / \mathrm{dia})^{(10)}$, que pode ser progressivamente reduzida até a retirada, usualmente controla a doença ${ }^{(7)}$. Se melhora significativa não ocorrer até o final da segunda semana ou se ocorrer recaída durante a diminuição do corticoesteróide, a ciclofosfamida oral $(2 \mathrm{mg} / \mathrm{kg} / \text { dia })^{(10)}$ deve ser adicionada ao tratamento $^{(7)}$. Se a função auditiva não for restaurada até $o$ final da décima segunda semana, o dano auditivo é considerado irreversível e o tratamento deve ser descontinuado ${ }^{(7)}$. Os pacientes que apresentam apenas efusão da orelha média, como a razão para a perda auditiva de condução, podem se beneficiar da remoção do fluido e da inserção de tubo de ventilação com melhora sustentada ${ }^{(12)}$.

A remissão ocorre em $75 \%$ dos pacientes com a terapia medicamentosa descrita, em três a seis meses ${ }^{(10)}$, porém mais da metade dos pacientes que entra em remissão desenvolverá recaídas subseqüentes ${ }^{(10)}$. Deve-se salientar que esse regime está associado a toxicidades graves e a escolha terapêutica atual depende da gravidade da doença, sendo razoável o tratamento com a ciclofosfamida na indução da remissão a curto prazo, seguido do uso de drogas menos tóxicas, como o metotrexato ou a azatioprina na manutenção ${ }^{(10)}$. 
Alternativamente, o metotrexate em combinação com corticoesteróides pode ser usado na indução da remissão da GW limitada sem envolvimento orgânico grave, poupando os pacientes dos efeitos colaterais da ciclofosfamida ${ }^{(13,14)}$. Outra estratégia menos tóxica é o uso da ciclofosfamida EV em pulsos mensais, porém este esquema está associado a maiores taxas de recaídas ${ }^{(10)}$.

A terapia alternativa com metotrexate não elimina e, possivelmente, não reduz o risco de pneumonia por Pneumocystis carinii (Pneumocystis jiroveci) ${ }^{(15)}$, portanto, independentemente da terapia utilizada na indução de remissão (ciclofosfamida ou metotrexate), os pacientes com GW devem receber profilaxia contra pneumonia por Pneumocystis carinii com sulfametoxazol-trimetoprim ou dapsona ${ }^{(13)}$. A pentamidina inalada mensalmente é uma alternativa para pacientes que apresentam efeitos adversos com o uso de sulfametoxazol-trimetoprim, porém o custo é elevado ${ }^{(15)}$.

A estenose subglótica pode responder de maneira incompleta à terapia imunossupressora padrão, necessitando de dilatação cirúrgica da via aérea associada à injeção intralesional de corticoesteróides ${ }^{(16)}$.

\section{REFERÊNCIAS}

1. Kaweski S, Jackson IT, Rego M: Wegener's granulomatosis in the head and neck área: a difficult diagnosis. Eur J Surg 22: 173-6, 1999.

2. Hartl DM, Aïdan P, Brugière O, Sterkers O: Wegener's granulomatosis presenting as a recurrence of chronic otitis media. Am J Otolaryngol 19 (1): 54-60, 1998.

3. Wegener F: Wegener's granulomatosis thoughts and observations of a pathologist. Eur Arch Otorhinolaryngol 247: 133-42, 1990.

4. Furtado RN, Maia MB, Azevedo E, Franco M, Andrade LE: Comprometimento renal e ocular como manifestações iniciais de granulomatose de Wegener. Rev Bras Reumatol 37 (6): 343-7, 1997.

5. Thornton MA, O'Sullivan TJ: Otological Wegener granulomatosis: a diagnostic dilemma. Clin Otolaryngol 25: 433-4, 2000.

6. Gomides AP, Rosário EJ, Borges HM, et al.: Perda auditiva neurossensorial em pacientes com granulomatose de Wegener: Relato de três casos e revisão de literatura. Rev Bras Reumatol 46 (3): 234-6, 2006.

7. Pereira DB, Amaral JL, Szajubok CM, et al.: Manifestações otorrinolaringológicas nas doenças reumáticas auto-imunes. Rev Bras Reumatol 46 (2): 118-25, 2006.

8. Rasmussen N: Management of the ear, nose, and throut manifestations of Wegener granulomatosis: an otorhinolaryngologist's perpective. Curr Opin Rheumatol 13: 3-11, 2001.

\section{CONCLUSÃO}

A paciente apresentou forma limitada da GW, com acometimento da orelha média com perda auditiva condutiva e estenose subglótica. O diagnóstico de GW foi aventado depois de um período de antibioticoterapia sem sucesso. A avaliação otorrinolaringológica foi de extrema importância para o diagnóstico correto da entidade clínica. De modo não usual, a paciente não apresentava provas de atividade inflamatória elevadas e o c-ANCA era negativo, tanto no quadro inicial quanto na recidiva. O diagnóstico foi possível com a análise do espécime histológico da membrana timpânica, em 2001. Provavelmente por causa do não-seguimento e da conseqüente falta de uma droga de manutenção, a paciente apresentou recaída após três anos assintomática. A presença de sintomas semelhantes ao quadro inicial, além do estridor e da estenose subglótica, bem como alterações em exames de imagem (RNM), ajudaram a caracterizar o segundo episódio de diminuição da acuidade auditiva como recidiva da doença. Reintroduzido o esquema terapêutico inicial, a paciente obteve melhora sintomática, porém mantendo certo grau de hipoacusia.

9. Macias JD, Wackym PA, McCabe BF: Early diagnosis of otologic Wegener's granulomatosis using the serologic marker c-ANCA. Ann Otol Rhinol Laryngol 102: 337-41, 1993.

10. Mabashir E, Ahmed MM, Hayat S, et al.: Wegener granulomatosis: a case report and update. South Med J 99 (9): 977-88, 2006.

11. Takagi D, Nakamaru Y, Maguchi S, et al.: Otologic manifestations of Wegener's granulomatosis. Laryngoscope 112: 1684-90, 2002.

12. Bakthavachalam S, Driver MS, Cox C, et al.: Hearing loss in Wegener's granulomatosis. Otol Neurotol 25: 833-7, 2004.

13. Stone JH. In: Imboden H, editor. Current rheumatology diagnosis and treatment. 2nd ed. Lange Medical Books/ McGraw-Hill; 2007.

14. De Groot K, Rasmussen N, Bacon PA, et al.: Randomized trial of cyclophosphamide versus methotrexate for induction of remission in early systemic antineutrophil cytoplasmic antibody - associated vasculitis. Arthritis Rheum 52 (8): 2461-9, 2005.

15. Chung JB, Armstrong K, Schwartz JS, Albert D: Costeffectiveness of prophylaxis against pneumocystis carinni pneumonia in patients with Wegener's granulomatosis undergoing immunosuppressive therapy. Arthritis Rheum 43 (8): $1841-8,2000$.

16. Hoffman GS, Thomas-Golbanov CK, Chan J, et al.: Treatment of subglottic stenosis due to Wegener's granulomatosis with intralesional corticosteroids and dilatation. J Rheumatol 30 (5): 1017-21, 2003. 